\title{
A VARIATIONAL METHOD FOR BOUNDARY VALUE PROBLEMS WITH DISCONTINUOUS NONLINEARITIES
}

\author{
C. A. STUART AND J. F. TOLAND
}

\section{Introduction}

Let $\Omega$ be a bounded domain in $\mathbb{R}^{n}$ which satisfies the cone property, and let $p \in L^{2}(\Omega)$. We study the boundary value problem

$$
\begin{aligned}
-\Delta u(x) & =f(u(x))+p(x), x \in \Omega \\
u(x) & =0, x \in \partial \Omega
\end{aligned}
$$

when $f$ is locally a function of bounded variation on $\mathbb{R}$. We do not suppose that $f$ is continuous, but only that $f(t)$ lies between $f(t+)$ and $f(t-)$ for all $t \in \mathbb{R}$.

Experience teaches that, in general, solutions of (1.1) need not exist even under the most restrictive hypotheses (see [4], [6] and [7]) and that it is wise to consider instead multi-valued versions of (1.1), for which an adequate existence theory obtains (see [7]).

The main results of this paper are contained in the existence theorems of Section 3 for multi-valued versions of (1.1), and they are obtained using a variational approach in Hilbert space. The situation under consideration is more or less the same as that of [7] but we find that our variational arguments give extra information which is unavailable by other means. We shall return to this later, but first we need to discuss what is meant by a variational formulation when $f$ is discontinuous.

The functional normally associated with (1.1) is

$$
\mathscr{J}(u)=\int_{\Omega}\left\{\frac{1}{2}|\nabla u(x)|^{2}-F(u(x))-p(x) u(x)\right\} d x,
$$

where $F$ is a primitive of $f$. But in our case, when $f$ may be discontinuous, $\mathscr{J}$ fails to be Fréchet differentiable, and the usual notion of critical point is no longer available.

To overcome this difficulty we decompose $f$ as $g-h$, the difference of two nondecreasing functions on $\mathbb{R}$ (and this can be done because we suppose that $f$ is locally of bounded variation). Then we define a function $\mathscr{H}-\mathscr{G}$ on a suitable Banach space $V$, by

$$
\mathscr{H}(u)-\mathscr{G}(u)=\int_{\Omega}\left\{\frac{1}{2}|\nabla u(x)|^{2}+H(u(x))\right\} d x-\int_{\Omega}\{G(u(x))+p(x) u(x)\} d x
$$

Received 5 February, 1979.

[J. LONDON Math. SoC. (2), 21 (1980), 319-328] 
Here $G$ and $H$ are primitives of $g$ and $h$ respectively and so both $\mathscr{G}$ and $\mathscr{H}$ are convex functionals on $V$. For such a functional $\mathscr{H}-\mathscr{G}$ there is a definition of critical point [9] which says that $u \in V$ is a critical point of $\mathscr{H}-\mathscr{G}$ if and only if

$$
\partial \mathscr{G}(u) \cap \partial \mathscr{H}(u) \neq \varnothing
$$

Here $\partial \mathscr{H}(u)$ and $\partial \mathscr{G}(u)$ denote respectively the set of sub-differentials of $\mathscr{H}$ and $\mathscr{G}$ at $u$ (for further details and references, see [9]).

It is clear that, in general, the fact that $u$ is a critical point of $\mathscr{H}-\mathscr{G}$ depends on $\mathscr{H}$ and on $\mathscr{G}$, rather than on the functional $I$ whose value at each point of $u \in V$ is given by $I(u)=\mathscr{H}(u)-\mathscr{G}(u)$. (For example, for a $C^{\infty}$-function $I$ of one variable it is possible to find $\mathscr{H}$ and $\mathscr{G}$, both convex functions of $\mathbb{R}$, such that $I(u)=\mathscr{H}(u)-\mathscr{G}(u)$ for all $u \in \mathbb{R}$, $\partial \mathscr{H}(\mathbf{u}) \cap \partial \mathscr{G}(\mathbf{u}) \neq \varnothing$ yet $I^{\prime}(\mathbf{u}) \neq 0$.)

In Section 2 we will show that, provided the decomposition of $f$ is restricted to an allowable class, every critical point of $\mathscr{H}-\mathscr{G}$ in $L^{2}(\Omega)$ is in fact in $W_{0}^{1,2}(\Omega) \cap W^{2,2}(\Omega)$ and satisfies (1.1) in the following multi-valued sense:

where

$$
-\Delta u(x)-p(x) \in \hat{f}(u(x)) \text { for almost all } x \in \Omega
$$

$$
\hat{f}(t)= \begin{cases}{[f(t-), f(t+)]} & \text { if } f(t-) \leqslant f(t+), \\ {[f(t+), f(t-)]} & \text { if } f(t+)<f(t-) .\end{cases}
$$

Indeed because of the Sobolev embedding theorems we shall see that, in fewer than 4 dimensions, there is a one-to-one correspondence between solutions of (1.2) and critical points of $\mathscr{H}-\mathscr{G}$, whatever admissible decomposition of $f$ we choose. In higher dimensions there exist solutions of (1.2) which are only critical points of $\mathscr{H}-\mathscr{G}$ for some decompositions of $f$ and not for others. An example in Section 4 makes this claim explicit.

In Section 3 we consider the question of the existence of critical points of $\mathscr{H}-\mathscr{G}$. We will suppose that $f$ satisfies the one-sided growth condition used in [7], namely

$$
\limsup _{|t| \rightarrow \infty} \frac{f(t)}{t}<\Lambda
$$

where $\Lambda$ is the first eigenvalue for the linear problem

$$
\begin{aligned}
-\Delta u(x) & =\lambda u(x) \text { on } \Omega, \\
u(x) & =0 \quad \text { on } \partial \Omega .
\end{aligned}
$$

Under these hypotheses we show that, for an arbitrary admissible decomposition of $f$ there exists a minimiser $\mathbf{u}$ of $\mathscr{H}-\mathscr{G}$ in $L^{\infty}(\Omega)$. It follows at once that $\partial \mathscr{G}(\mathbf{u}) \neq \varnothing$ and from Theorem 2.3 of [9] that $\mathbf{u}$ is a critical point of $\mathscr{H}-\mathscr{G}$. Thus u satisfies (1.2).

But Theorem 2.3 of [9] says more than that $\mathbf{u}$ is a critical point of $\mathscr{H}-\mathscr{G}$; it says that $\partial \mathscr{G}(\mathbf{u}) \subseteq \partial \mathscr{H}(\mathbf{u})$ (a much stronger claim than that $\partial \mathscr{H}(\mathbf{u}) \cap \partial \mathscr{G}(\mathbf{u}) \neq \varnothing)$. From this we show that the set

$$
\{x: f(\mathbf{u}(x)+)>f(\mathbf{u}(x)-)\}
$$


has zero measure. Thus $\mathbf{u}$ satisfies (1.2), but even more is true; $u \in W_{0}^{1,2}(\Omega) \cap W^{2,2}(\Omega) \cap L^{\infty}(\Omega)$ and

$$
-\Delta u(x)-p(x) \in \tilde{f}(u(x)) \text { almost everywhere on } \Omega
$$

where

$$
\tilde{f}(t)= \begin{cases}\{f(t)\} & \text { if } f(t+) \geqslant f(t-), \\ \hat{f}(t) & \text { if } f(t-)>f(t+) .\end{cases}
$$

In the last section we give two examples. In one we see how a solution of (1.2) may or may not be a critical point of $\mathscr{H}-\mathscr{G}$ depending on the decomposition of $f$ chosen.

In the other we show that (1.4) does not hold for all solutions of (1.5) (or indeed (1.1)). It is however always a property of a minimiser of $\mathscr{H}-\mathscr{G}$ if $(1.3)$ holds. Thus the existence of examples such as the one with which we finish often means that a non-uniqueness result holds for solutions of (1.5). This observation is further elaborated in a forthcoming paper [8].

Finally it is worth remarking that the classical variational approach to semi-linear elliptic problems may fail, not because $f$ is discontinuous, but because we lack suitable growth behaviour of $f$ at infinity. Our method of attack proves equally useful then.

\section{The variational formulation}

Let $B$ denote the set of all functions $f: \mathbb{B} \rightarrow \mathbb{R}$ which have bounded variation on compact intervals. If $f \in B$ then for all $t \in \mathbb{R}, f(t \pm)=\lim _{\varepsilon \rightarrow 0+} f(t \pm \varepsilon)$ exists. Define the upward-jump-set $(U(f))$ and the downward-jump-set $(D(f))$ to be

$$
\begin{aligned}
& U(f)=\{t \in \mathbb{R}: f(t-)<f(t+)\}, \\
& D(f)=\{t \in \mathbb{R}: f(t+)<f(t-)\} .
\end{aligned}
$$

Then for $f \in B, f$ is continuous except on the set $D(f) \cup U(f)$. Put

and

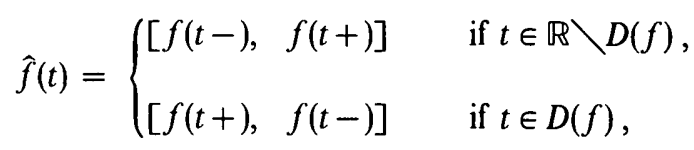

$$
\tilde{f}(t)= \begin{cases}\{f(t)\} & \text { if } t \in \mathbb{R} \backslash D(f), \\ {[f(t+), \quad f(t-)]} & \text { if } t \in D(f) .\end{cases}
$$

Thus $\hat{f}$ is a multi-valued mapping on $\mathbb{R}$ which fills in all the jumps of $f$, whereas $\hat{f}$ only fills in the downward jumps of $f$. Let

$$
\mathrm{BN}=\{f \in B: f(t) \in \hat{f}(t) \text { for all } t \in \mathbb{R}\}
$$

The class BN was introduced in [7] and its usefulness for the problem (1.1) depends on the following result which involves a slight extension of Lemma 2.2 of [7]. 
LEMma 2.1. Let $f \in \mathrm{BN}$. Then there exist two increasing functions $g: \mathbb{R} \rightarrow \mathbb{R}$ and $h: \mathbb{R} \rightarrow \mathbb{R}$ which have the following properties:

(i) $f(t)=g(t)-h(t)$ for all $t \in \mathbb{R}$;

(ii) $g$ is continuous on $\mathbb{R} \backslash U(f)$;

(iii) $h$ is continuous on $\mathbb{R} \backslash D(f)$;

(iv) $\hat{f}(t)=\hat{g}(t)-\hat{h}(t)$ and $\hat{f}(t)=g(t)-\hat{h}(t)$ for all $t \in \mathbb{R}$.

Remark. Clearly a decomposition with these properties is only unique up to the addition of continuous increasing functions to $g$ and $h$. We shall call a decomposition of $f$, as the difference of two functions $g-h$, admissible if and only if $g$ and $h$ satisfy all the properties of Lemma 2.1, and $g(0)=0$.

Hence we shall only consider $f \in \mathrm{BN}$, and those $g, h$, whose difference is an admissible decomposition of $f$. Let

$$
G(t)=\int_{0}^{t} g(s) d s \quad \text { and } \quad H(t)=\int_{0}^{t} h(s) d s \text { for all } t \in \mathbb{R}
$$

Then both $G$ and $H$ are convex, continuous functions on $\mathbb{R}$. For each $u \in L^{2}(\Omega)$ we shall define extended-real-valued functionals $\mathscr{G}$ and $\mathscr{H}$ as follows:

$$
\begin{aligned}
& \mathscr{H}(u)= \begin{cases}\int_{\Omega}\left\{\frac{1}{2}|\nabla u(x)|^{2}+H(u(x))\right\} d x & \text { if } u \in W_{0}^{1,2}(\Omega), H(u) \in L^{1}(\Omega), \\
+\infty & \text { otherwise; }\end{cases} \\
& \mathscr{G}(u)= \begin{cases}\int_{\Omega}\{G(u(x))+p(x) u(x)\} d x & \text { if } G(u) \in L^{1}(\Omega), \\
+\infty & \text { otherwise. }\end{cases}
\end{aligned}
$$

Lemma 2.2. Both $\mathscr{G}$ and $\mathscr{H}: L^{2}(\Omega) \rightarrow \mathbb{B} \cup\{+\infty\}$ are convex and lower semicontinuous.

(i) An element $v \in L^{2}(\Omega)$ belongs to the subdifferential of $\mathscr{G}$ at $u$ if and only if $v(x) \in \hat{g}(u(x))+p(x)$ for almost all $x \in \Omega$.

(ii) An element $v \in L^{2}(\Omega)$ belongs to the subdifferential of $\mathscr{H}$ at $u$ if and only if $u \in W_{0}^{1,2}(\Omega) \cap W^{2,2}(\Omega)$, and $v(x) \in-\Delta u(x)+\widehat{h}(u(x))$ for almost all $x \in \Omega$.

(iii) For each $u \in L^{2}(\Omega)$, the set $\partial \mathscr{G}(u) \cap \partial \mathscr{H}(u)$ is either empty, or contains a single element.

Proof. The convexity and lower semi-continuity results and parts (i) and (ii) are standard (see, for example [1], or [2; Proposition 2.8 and Proposition 3.8]). 
(iii) Suppose that $v, w \in \partial \mathscr{G}(u) \cap \partial \mathscr{H}(u)$. Then

$$
\begin{aligned}
& u \in W_{0}^{1,2}(\Omega) \cap W^{2,2}(\Omega), \\
& v(x), w(x) \in \hat{g}(u(x))+p(x), \\
& v(x), w(x) \in-\Delta u(x)+\hat{h}(u(x)),
\end{aligned}
$$

for all $x \in E$, where $E \subseteq \Omega$ and meas. $(E)=$ meas. $(\Omega)$.

If $x \in E$, then either $u(x) \in U(f)$, in which case $h$ is continuous at $u(x)$, and this implies that

$$
v(x)=w(x)=-\Delta u(x)+h(u(x))
$$

or $u(x) \in \mathbb{R} \backslash U(f)$ and $g$ is continuous at $u(x)$, and this implies that

$$
v(x)=w(x)=g(u(x))+p(x) .
$$

Thus $v(x)=w(x)$ for all $x \in E$ and so almost everywhere in $\Omega$. Hence $v=w$ in $L^{2}(\Omega)$. if

Now according to [9] a point $u \in L^{2}(\Omega)$ is called a critical point for $\mathscr{H}-\mathscr{G}$ if and only

$$
\partial \mathscr{G}(u) \cap \partial \mathscr{H}(u) \neq \varnothing .
$$

Because of our definition of admissible decomposition of $f$ we have the following result.

THEOREM 2.3. Let $f \in \mathrm{BN}$ and let $g-h$ be an admissible decomposition of $f$. An element $u \in L^{2}(\Omega)$ is a critical point of $\mathscr{H}-\mathscr{G}$ if and only if

(i) $u \in W_{0}^{1,2}(\Omega) \cap W^{2,2}(\Omega)$;

(ii) $[1-\chi(u)] g(u) \in L^{2}(\Omega)$;

(iii) $\chi(u) h(u) \in L^{2}(\Omega)$; and

(iv) $-\Delta u(x)-p(x) \in \hat{f}(u(x))$ for almost all $x \in \Omega$.

Here $\chi(t)=1$ if $t \in U(f)$, and $\chi(t)=0$ otherwise.

Remark. One might feel that the critical points of $\mathscr{H}-\mathscr{G}$ should be independent of the decomposition $f=g-h$. That this is not the case is clear from an example in Section 4. However it follows from the above characterisation that, if $u$ is a critical point of $\mathscr{H}-\mathscr{G}$ and $u \in L^{\infty}(\Omega)$, then it is a critical point for every admissible decomposition of $f$. This fact is exploited in the analysis in Section 3.

Proof. If $u \in L^{2}(\Omega)$ is a critical point of $\mathscr{H}-\mathscr{G}$, then $u \in W_{0}^{1,2}(\Omega) \cap W^{2,2}(\Omega)$ and there exists $v \in L^{2}(\Omega)$ such that

and

$$
v(x) \in \hat{g}(u(x))+p(x),
$$

$$
v(x) \in-\Delta u(x)+\hat{h}(u(x)),
$$


for all $x \in E \subseteq \Omega$ with meas. $(E)=$ meas. $(\Omega)$. Thus

and

$$
[1-\chi(u(x))] v(x)=[1-\chi(u(x))][g(u(x))+p(x)]
$$

$$
\chi(u(x)) v(x)=\chi(u(x))[-\Delta u(x)+h(u(x))]
$$

for all $x \in E$. Thus (i), (ii) and (iii) are established. Moreover we have also that

and

$$
\chi(u(x)) v(x) \in \chi(u(x))[\hat{g}(u(x))+p(x)]
$$

$$
[1-\chi(u(x))] v(x) \in[1-\chi(u(x))][-\Delta u(x)+\hat{h}(u(x))] .
$$

Therefore when $x \in E$ and $\chi(u(x))=1$,

$$
v(x)=-\Delta u(x)+h(u(x)) \in \hat{g}(u(x))+p(x) .
$$

When $x \in E$ and $\chi(u(x))=0$,

$$
v(x)=g(u(x))+p(x) \epsilon-\Delta u(x)+\hat{h}(u(x)) .
$$

Thus for all $x \in E$

$$
-\Delta u(x)-p(x) \in \hat{f}(u(x))
$$

and (iv) is established. Conversely suppose that (i), (ii), (iii), (iv) hold. Then

$$
-\Delta u(x)-p(x) \in[1-\chi(u(x))][g(u(x))-\hat{h}(u(x))]+\chi(u(x))[\hat{g}(u(x))-h(u(x))]
$$

almost everywhere on $\Omega$. Let

$$
v(x)=[1-\chi(u(x))][g(u(x))+p(x)]+\chi(u(x))[-\Delta u(x)+h(u(x))] .
$$

Then

$$
\begin{aligned}
& v \in L^{2}(\Omega), \\
& v(x) \in \hat{g}(u(x))+p(x), \text { and } \\
& v(x) \in-\Delta u(x)+\hat{h}(u(x)) \text { for almost all } x \in \Omega .
\end{aligned}
$$

Thus $v \in \partial \mathscr{G}(u) \cap \partial \mathscr{H}(u)$, by Lemma 2.2, and the proof of the theorem is complete.

Corollary 2.4. If $f \in \mathrm{BN}$ has an admissible decomposition which is such that $g(u) \in L^{2}(\Omega)$ for all $u \in W_{0}^{1,2}(\Omega) \cap W^{2,2}(\Omega)$, then $u$ is a critical point of $\mathscr{H}-\mathscr{G}$ if and only if $u \in W_{0}^{1,2}(\Omega) \cap W^{2,2}(\Omega)$, and $-\Delta u(x)-p(x) \in \hat{f}(u(x))$ a.e. on $\Omega$.

Proof. Suppose that $u \in W_{0}^{1,2}(\Omega) \cap W^{2,2}(\Omega)$, and that $-\Delta u(x)-p(x) \in \hat{f}(u(x))$. It then follows from the hypotheses that $[1-\chi(u)] g(u) \in L^{2}(\Omega)$. Thus, according to Theorem $2.3, u$ is a critical point of $\mathscr{H}-\mathscr{G}$ if we can show that $\chi(u) h(u) \in L^{2}(\Omega)$. It suffices to show that if $v(x) \in \hat{g}(u(x))$ for almost all $x \in \Omega$, then $v \in L^{2}(\Omega)$. 
But $g(0)=0$ and $g$ is increasing. Therefore $|v(x)|<K+|g(2 u(x))|$ for almost all $x \in \Omega$, where $K=g(0+)-g(0-)$. Hence $v \in L^{2}(\Omega)$, and $u$ is a critical point of $\mathscr{H}-\mathscr{G}$.

Conversely if $u$ is a critical point of $\mathscr{H}-\mathscr{G}$, then the conclusion follows at once from Theorem 2.3.

Corollary 2.5. For $n=1,2,3$ and for every admissible decomposition of $f$ there is a one-to-one correspondence between critical points of $\mathscr{H}-\mathscr{G}$ and solutions of (1.2) in $W^{2,2}(\Omega) \cap W_{0}^{1,2}(\Omega)$.

For $n=4$, if $f$ admits a decomposition with $g$ satisfying the growth condition

$$
|g(p)|<M+|p|^{\alpha} \text { for } M>0, \alpha \in \mathbb{R},
$$

or if $n \geqslant 5$ and $f$ admits a decomposition with $g$ satisfying

$$
|g(p)|<M+|p|^{\alpha}, \quad M>0, \quad \alpha<\left(\frac{n}{n-4}\right),
$$

then there is a one-to-one correspondence between the criticial points of $\mathscr{H}-\mathscr{G}$ (for this choice of decomposition) and solutions of (1.2) in $W^{2,2}(\Omega) \cap W_{0}^{1,2}(\Omega)$.

Proof. This follows at once from the previous corollary, and the standard Sobolev embedding theorems of $W^{2,2}(\Omega)$ in $L^{2}(\Omega)$ which hold since $\Omega$ satisfies the cone condition (see, e.g. [3]).

Our next result gives emphasis to a certain type of critical point and derives its interest from the fact that all minimisers of $\mathscr{H}-\mathscr{G}$ are of this type (see Theorem 3.1 of Section 3).

THEOREM 2.6. Let $f$ have an admissible decomposition as $g-h$ and let $\mathbf{u}$ be a critical point of $\mathscr{H}-\mathscr{G}$. If, moreover, $\partial \mathscr{G}(\mathbf{u}) \subseteq \partial \mathscr{H}(\mathbf{u})$ then $\chi(\mathbf{u}(x))=0$ for almost all $x \in \Omega$, and consequently,

$$
-\Delta \mathbf{u}(x)-p(x) \in \widetilde{f}(\mathbf{u}(x)) \text { for almost all } x \in \Omega
$$

Proof. It suffices to show that $\chi(\mathbf{u}(x))=0$ for almost all $x \in \Omega$. So let $v \in \partial \mathscr{G}(\mathbf{u})$ and, for any $t \in U(f)$, set

where

$$
w_{ \pm}(x)=\left[1-\chi_{t}(\mathbf{u}(x))\right] v(x)+\chi_{t}(\mathbf{u}(x))[g(t \pm)+p(x)]
$$

$$
\chi_{t}(s)= \begin{cases}1 & \text { if } s=t \\ 0 & \text { otherwise }\end{cases}
$$

Then $w_{ \pm}$belong to $L^{2}(\Omega)$ and $w_{ \pm}(x) \in \hat{g}(\mathbf{u}(x))+p(x)$ for almost all $x \in \Omega$. So $w_{ \pm} \in \partial \mathscr{G}(\mathbf{u})$, and hence $w_{ \pm} \in \partial \mathscr{H}(\mathbf{u})$. It now follows from Lemma 2.2(iii) that $w_{+}(x)=w_{-}(x)=v(x)$ for almost all $x \in \Omega$, and so $\chi_{t}(\mathbf{u}(x))=0$ for almost all $x \in \Omega$. Since $U(f)$ is countable, and

$$
\chi(\mathbf{u}(x))=\sum_{t \in U(f)} \chi_{t}(\mathbf{u}(x))
$$

it follows that $\chi(\mathbf{u}(x))=0$ almost everywhere. 
Remark. That $\chi(\mathbf{u}(x))=0$ almost everywhere means that $\mathbf{u}$ takes values in the upward-jump-set of $f$ on a set of zero measure.

\section{On minimisers of $\mathscr{H}-\mathscr{G}$}

Our considerations in this section concern the existence of solutions of the boundary value problem (1.5) under the same hypotheses as in [7]. The following observation is central to the analysis.

Theorem 3.1. Let $\mathscr{H}$ and $\mathscr{G}$ be convex lower semi-continuous functionals from a Banach space $V$ into $\mathbb{R} \cup\{+\infty\}$. If $\mathbf{u}$ is a minimiser of $\mathscr{H}-\mathscr{G}$ in $V$, then

$$
\partial \mathscr{G}(\mathbf{u}) \subseteq \partial \mathscr{H}(\mathbf{u})
$$

Proof. This is Theorem 2.3 of [9].

THEOREM 3.2. Let $f$ satisfy (1.3) and let $g-h$ be any admissible decomposition for $f$. Then there exists a minimiser $\mathbf{u}$ of $\mathscr{H}-\mathscr{G}$ which is in $W_{0}^{1.2}(\Omega) \cap W^{2,2}(\Omega) \cap L^{\infty}(\Omega)$ and which satisfies

$$
-\Delta \mathbf{u}(x)-p(x) \in \tilde{f}(\mathbf{u}(x))
$$

and $\chi(\mathbf{u}(x))=0$ almost everywhere in $\Omega$. It follows that $\mathbf{u}$ is a minimiser of $\mathscr{H}-\mathscr{G}$ for all possible admissible decompositions of $f$.

Proof. Let $g-h$ be an admissible decomposition for $f$. Then by (1.3) there exist constants $\varepsilon, M>0$ such that

and

$$
f(t) \leqslant M+(\Lambda-\varepsilon) t \text { for all } t>0
$$

Hence

$$
f(t) \geqslant(\Lambda-\varepsilon) t-M \text { for all } t>0 .
$$

$$
F(t) \leqslant \frac{1}{2}(\Lambda-\varepsilon) t^{2}+M|t| \text { for all } t \in \mathbb{R} .
$$

Now $F(t)=G(t)-H(t)$ and hence it follows that for all $u \in L^{2}(\Omega)$,

$$
\mathscr{J}(u)=\int_{\Omega}\left\{\frac{1}{2}|\nabla u(x)|^{2}-p(x) u(x)-F(u(x))\right\} d x \leqslant \mathscr{H}(u)-\mathscr{G}(u)
$$

Note that equality need not hold since $G(u), H(u)$ need not be integrable.

We will prove that there exists an $L^{\infty}$-minimiser $\mathbf{u}$ of $\mathscr{J}$ in $L^{2}(\Omega)$, so in consequence

$$
\mathscr{J}(\mathbf{u})=\mathscr{H}(\mathbf{u})-\mathscr{G}(\mathbf{u})
$$

Therefore $\mathbf{u}$ is a minimiser for $\mathscr{H}-\mathscr{G}$ no matter which decomposition of $f$ we choose. 
If $\varepsilon$ and $M$ are chosen as above, then

$$
\begin{aligned}
\mathscr{J}(u) \geqslant & \int_{\Omega}\left\{\frac{1}{2}|\nabla u(x)|^{2}-p(x) u(x)-\frac{1}{2}(\Lambda-\varepsilon) u(x)^{2}-M|u(x)|\right\} d x \\
& \geqslant \int_{\Omega}\left\{\frac{\varepsilon}{2 \Lambda}|\nabla u(x)|^{2}-(|p(x)|+M)|u(x)|\right\} d x \text {, since } \\
& \int_{\Omega}|\nabla u(x)|^{2} d x \leqslant \Lambda \int_{\Omega}|u(x)|^{2} d x \text { for all } u \in W_{0}^{1,2} .
\end{aligned}
$$

Hence $\mathscr{J}$ is coercive in $W_{0}^{1,2}(\Omega)$, and bounded below, and it follows that any minimising sequence $\left\{u_{n}\right\}$ has a subsequence (which we also denote by $\left\{u_{n}\right\}$ ) which is weakly convergent to $\mathrm{u}$, say, in $W_{0}^{1,2}$. But,

$$
F(t) \leqslant(\Lambda-\varepsilon) t^{2}+M|t|,
$$

and it then follows by Fatou's lemma that $\mathscr{J}$ is lower semi-continuous in the sense that when $u_{n} \rightarrow \mathbf{u}$ in $W_{0}^{1,2}$

$$
\liminf _{n \rightarrow \infty} \mathscr{J}\left(u_{n}\right) \geqslant \mathscr{J}(\mathbf{u}) .
$$

Thus $\mathbf{u}$ is a minimiser for $\mathscr{J}$.

Finally to prove $\mathbf{u} \in L^{\infty}(\Omega)$ we call upon a result of [5]. Putting $G_{1}(t)=\frac{1}{2} \Lambda t^{2}-F(t)$ and $G_{2}(t)=-\frac{1}{2} \Lambda t$, it follows from Theorem 6.2 of [5] that $u \in L^{\infty}(\Omega)$. In [5] $G_{1}$ and $G_{2}$ are required to be $C^{1}$, but it is easy to see that the result holds in our case as well. The rest of the proof follows by Theorems 2.6 and 3.1.

\section{Two examples}

(i) Let us consider $\Omega$ to be the unit ball in $\mathbb{R}^{5}$, and let us consider the boundary value problem

$$
\begin{aligned}
-\Delta u(x) & =\frac{8}{9}(u(x)+1)^{7} \\
u(x) & =0, \quad x \in \partial \Omega .
\end{aligned}
$$

Then the radially symmetric function

$$
\mathbf{u}(x)=\left(|x|^{-1 / 3}-1\right)
$$

is an exact solution which lies in $W^{2,2}(\Omega) \cap W_{0}^{1,2}(\Omega)$, but not in $L^{\infty}(\Omega)$.

Hence if we put $g(t)=\frac{8}{9}(t+1)^{7}$ and $h=0$ we find that $\mathbf{u}$ is a critical point of $\mathscr{H}-\mathscr{G}$, 
but if we write $g(t)=\frac{8}{9}(t+1)^{7}+\exp t$, and $h(t)=\exp t$, then $\mathbf{u}$ is no longer a critical point of $\mathscr{H}-\mathscr{G}$ for this new decomposition.

(ii) For each $\varepsilon \in[-1,1]$ define the function

$$
f_{\varepsilon}(t)=\left\{\begin{aligned}
-1 & \text { if } t<0 \\
\varepsilon & \text { if } t=0 \\
+1 & \text { if } t>0
\end{aligned}\right.
$$

Then $f_{\varepsilon}$ is in $\mathrm{BN}$ and satisfies the hypotheses (1.3). Thus there always exists a minimiser of $\mathscr{H}-\mathscr{G}$ in $L^{\infty}(\Omega)$, no matter what decomposition of $f_{\varepsilon}$ we choose. For all $\varepsilon \in[-1,1]$ the function $u \equiv 0$ is a solution of

$$
-\Delta u(x) \in \hat{f}_{\varepsilon}(u(x))
$$

but only when $\varepsilon=0$ is it a solution of

$$
-\Delta u(x) \in \tilde{f}(u(x)) .
$$

In either case $\{x: f(u(x)+)>f(u(x)-)\}$ has full measure, and so 0 is not the minimiser of $\mathscr{H}-\mathscr{G}$. Thus such solutions immediately guarantee a non-uniqueness result. This idea is further developed in [8], where the question of what happens to the zero solution of $-\Delta u(x)=f_{\varepsilon}(u(x))$ almost everywhere when $\varepsilon$ is changed from zero is also examined more closely.

\section{References}

1. H. Brézis, "Monotonicity methods in Hilbert space and some applications to nonlinear partial differential equations", Contributions to nonlinear functionals analysis (ed. E. H. Zarantonello, M.R.C. Wisconsin, Academic Press, New York, 1971).

2. V. Barbu, Nonlinear semigroups and differential equations in Banach space (Noordhoff International Publishing, Leyden, 1976).

3. A. Friedman, Elliptic partial differential equations (Holt Reinhart and Winston, New York, 1969).

4. I. Massabo and C. A. Stuart, "Elliptic eigenvalue problems with discontinuous non-Iinearities', J. Math. Anal. Appl., 66 (1978), 261-281.

5. G. Stampacchia, "On some regular multiple integral problems in the calculus of variations", Comm. Pure Appl. Math., 16 (1963), 383-421.

6. C. A. Stuart, "Differential equations with discontinuous non-linearities", Arch. Rational Mech. Anal., 63 (1976), 59-75.

7. C. A. Stuart, "Maximal and minimal solutions of differential equations with discontinuous nonlinearities", Math. Z., 163 (1978), 239-249.

8. C. A. Stuart and J. F. Toland, "A property of solutions of elliptic differential equations with discontinuous nonlinearities", J. London Math. Soc. (2), 21 (1980), 329-335.

9. J. F. Toland, "A duality principle for non-convex optimisation and the calculus of variations", Arch. Rational Mech. Anal., 71 (1979), 41-61.

Département de Mathématique,

École Polytechnique Fédérale de Lausanne,

6, av. de Cour,

Lausanne $\mathrm{CH}-1007$,

Switzerland.
Department of Mathematics, University College, Gower Street, London WC1E 6BT. 